\title{
Volumetría hipocampal: análisis comparativo de los métodos de evaluación en enfermedad de Alzheimer
}

\section{Hippocampal Volumetry: Comparative Analysis of Evaluation Methods in Alzheimer's Disease}

\author{
Mauricio Franco Farez ${ }^{4,5}$ Paulina Yañez ${ }^{1}$ \\ ${ }^{1}$ Departamento de Diagnóstico por Imágenes, Instituto de Investigaciones \\ Neurológicas Dr. Raúl Carrea (FLENI), Buenos Aires, Argentina \\ 2 Laboratorio de Neuroimágenes, Instituto de Investigaciones \\ Neurológicas Dr. Raúl Carrea (FLENI), Buenos Aires, Argentina \\ 3 Servicio de Neurología Cognitiva, Neuropsicología y Neuropsiquiatría, \\ Departamento de Neurología, Instituto de Investigaciones Neurológicas \\ Dr. Raúl Carrea (FLENI), Buenos Aires, Argentina \\ ${ }^{4}$ Centro de Epidemiología, Bioestadística y Salud Pública (CEBES), \\ Buenos Aires, Argentina \\ ${ }^{5}$ Centro para la Investigación de Enfermedades Neuroinmunológicas \\ (CIEN), del Instituto de Investigaciones Neurológicas Dr. Raúl Carrea \\ (FLENI), Buenos Aires, Argentina
}

Hernán Chaves ${ }^{1,2}$ Cecilia Eugenia Rollan ${ }^{1}$ Fernando Ventrice ${ }^{2, \Omega} \quad$ Ismael Luis Calandri ${ }^{3}$

\begin{abstract}
Address for correspondence Hernán Chaves, MD, Departamento de Diagnóstico por Imágenes, Instituto de Investigaciones Neurológicas Dr. Raúl Carrea (FLENI), Montañeses 2325 (C1428AQK) CABA, Buenos Aires, Argentina (e-mail: hchaves@fleni.org.ar).
\end{abstract}

Rev Argent Radiol 2018;82:57-63.

\section{Resumen \\ Palabras Clave \\ - enfermedad de alzheimer \\ - biomarcadores \\ - imágenes de resonancia magnética \\ - hipocampos}

Objetivo La atrofia hipocampal es uno de los biomarcadores radiológicos más sensibles de la enfermedad de Alzheimer (EA) y existen diferentes métodos para evaluarla: análisis subjetivo visual (ASV), análisis objetivo manual (AOM) y análisis objetivo automático (AOA). Nos proponemos comparar esos métodos, y evaluar si el AOA presenta una confiabilidad cercana al AOM (método de referencia) y superior al ASV.

Materiales y Métodos Se seleccionaron retrospectivamente imágenes de resonancia magnética (RM) fast spoiled gradient-echo (FSPGR) de 28 sujetos ( 14 con deterioro cognitivo leve, 7 con EA y 7 controles). El ASV fue realizado por 10 radiólogos, clasificando la atrofia hipocampal en: nula, leve, moderada o severa. El AOM se basó en la segmentación manual de los hipocampos por dos operadores. El AOA fue realizada por medio del software FreeSurfer 5.3. Se calcularon coeficientes de correlación rho de Spearman para las variables discretas y coeficientes de correlación intraclase para las variables continuas.

Resultados Los coeficientes de correlación entre los dos operadores que realizaron el AOM fueron de $0,88(p<0,0001)$ para los hipocampos izquierdos y de 0,86 $(p<0,0001)$ para los hipocampos derechos. El coeficiente de correlación entre todos los ASV (promediados) y AOM fue de $-0,81$ (IC 95\% $-0,96--0,66$ ). Los coeficientes de correlación entre el AOA y el AOM fue de $0,54(p<0,0001)$ para los hipocampos izquierdos y de $0,61(p<0,0001)$ para los hipocampos derechos.

\footnotetext{
$\Omega$ Actualmente no afiliado al Instituto de Investigaciones Neurológicas Dr. Raúl Carrea (FLENI).
}

received

December 13, 2017

accepted

March 13, 2018

published online

June 1, 2018
DOI https://doi.org/

10.1055/s-0038-1656522. ISSN 1852-9992.
Copyright $\odot$ 2019, Sociedad Argentina de Radiología. Publicado por Thieme Revinter Publicações Ltda., Rio de Janeiro, Brazil. Todos los derechos reservados.
License terms

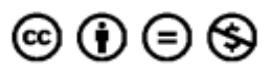




\author{
Abstract \\ Keywords \\ - alzheimer disease \\ - biomarkers \\ - magnetic resonance \\ imaging \\ - hippocampus
}

Conclusión Si bien el AOA tiene moderada correlación con el método de referencia, no es superior al ASV promedio y se deberían tomar recaudos antes de ser implementado en la práctica asistencial.

Objective Hippocampal atrophy is one of the most sensible radiological biomarkers of Alzheimer's disease. There are different methods to evaluate atrophy: visual subjective analysis (VSA), manual objective analysis (MOA) and automatic objective analysis (AOA). We will compare these methods and evaluate if AOA has a confidence similar to MOA (gold standard), and better than VSA.

Materials and Methods We retrospectively selected 3D FSPGR MRI from 28 subjects of whom 14 had mild cognitive impairment, 7 Alzheimer's disease and 7 controls. VSA was performed by 10 radiologists who classified hippocampal atrophy in none, mild, moderate and severe. For MOA, two operators manually segmented both hippocampus from all subjects. AOA was performed by FreeSurfer 5.3. Spearman's rho correlation coefficient was calculated for discrete variables and intraclass correlation coefficient was calculated for continuous variables.

Results Correlation coefficients between the operators that performed MOA was $0.88(p<0.0001)$ for left hippocampi and $0.86(p<0,0001)$ for right hippocampi. Correlation coefficients between mean VSA and MOA was $-0,81$ ( $95 \% \mathrm{Cl}-0,96--0,66)$. Correlation coefficients between AOA and AOM was $0.54(p<0.0001)$ for left hippocampi and $0.61(p<0.0001)$ for right hippocampi.

Conclusion Even though AOA has moderate correlation with the gold standard it is not superior to average VSA and should be implemented with care in clinical practice.

\section{Introducción}

Dentro de cualquier servicio de neuroradiología, una significativa cantidad de pacientes son evaluados por signos y/o síntomas de deterioro cognitivo. De ellos, un gran porcentaje padecen enfermedad de Alzheimer (EA) o su estadio clínico temprano, deterioro cognitivo leve (DCL), caracterizado fundamentalmente por fallas mnésicas anterógradas. La atrofia hipocampal es uno de los biomarcadores radiológicos más sensibles del conjunto EA-DCL. ${ }^{1,2}$

Existen diferentes formas de evaluar la atrofia hipocampal. Describiremos aquí, cuatro metodologías a las que llamaremos análisis subjetivo visual (ASV), análisis semiobjetivo visual (ASOV), análisis objetivo manual (AOM) y análisis objetivo automático (AOA). Cada una de esas técnicas tiene sus fortalezas y limitaciones.

El ASV es el método que habitualmente se utiliza para la valoración de la atrofia hipocampal en el ambiente clínico, utilizándose graduaciones poco comparables. Esa metodología, implica la inspección visual de los hipocampos en diferentes planos (principalmente el coronal), intentando mensurar el volumen del hipocampo sin tomar medidas ni utilizando escalas, en base al conocimiento previo y la experiencia del observador.

El ASOV implica la utilización de escalas previamente desarrolladas para clasificar el grado de atrofia hipocampal según diferentes características. La escala más utilizada es la elaborada por Scheltens y colaboradores, para la evaluación de la atrofia del lóbulo temporal mesial. Dicha graduación evalúa tres características (el ancho de la fisura coroidea, el ancho del asta temporal del ventrículo lateral y la altura de la formación hipocampal), obteniéndose una graduación de 0 (sin atrofia) a 4 (atrofia severa) según las características observadas. Decidimos no utilizar esa escala, ya que si bien ha demostrado su utilidad en estudios de investigación, habitualmente no es utilizada en la práctica radiológica clínica. $^{3}$

El AOM es la técnica de imágenes considerada como método de referencia, con buena correlación radiológicopatológica. ${ }^{4}$ Esa técnica precisa de entrenamiento y tiempo, ya que requiere de la delimitación de la formación hipocampal corte a corte, realizada por un operador, para luego obtener el volumen total segmentado. La metodología más aceptada y utilizada para realizar la segmentación manual es la definida por el Harmonized Protocol for Hippocampal Segmentation (HPHS), ha sido validada con gran estabilidad y reproducibilidad inter e intra operador. ${ }^{5-7}$

En los últimos años se han desarrollado diversos softwares que permiten un AOA, delimitando y obteniendo la volumetría de los hipocampos basados en atlas, sin requerimiento de un operador. ${ }^{8}$ Esa última técnica aún requiere de la validación con la segmentación manual.9,10

Nos proponemos comparar esos métodos para la determinación del volumen hipocampal (ASV y AOA), con respecto al actual método de referencia (AOM). Nuestro objetivo es evaluar si el AOA presenta una confiabilidad 
cercana al método de referencia y superior al ASV, siendo plausible de ser implementado en la práctica clínica.

\section{Materiales y Métodos}

Se escogieron retrospectivamente 28 sujetos participantes del estudio internacional multicéntrico Alzheimer's Disease Neuroimaging Initiative (ADNI), los cuales habían sido evaluados en nuestra institución en el período comprendido entre enero de 2012 y julio de 2013. Dicho protocolo, incluye el seguimiento con estudios de laboratorio (marcadores serológicos y en líquido cefalorraquídeo), estudios genéticos, tests cognitivos y estudios de imágenes de resonancia magnética (RM) y tomografía por emisión de positrones (PET). ${ }^{11}$ El protocolo ADNI y el presente trabajo están aprobados por el comité de ética de nuestra institución.

Los sujetos seleccionados (11 hombres y 17 mujeres), presentaban un rango etario de 65 a 84 años (media: 73,6 años; desvío estándar: 5,63 años) y se encontraban caracterizados clínicamente con deterioro cognitivo leve temprano (DCLt) $(n=7)$, con deterioro cognitivo leve avanzado (DCLa) $(n=7)$, con EA $(n=7)$ y sin deterioro cognitivo $(n=7)$. Se decidió incorporar igual cantidad de sujetos dentro de cada grupo para asegurarnos de una muestra clínicamente heterogénea. Sin embargo, esos sujetos fueron seleccionados en forma aleatoria hasta completar el cupo de cada grupo. La - Tabla 1 presenta información adicional de los sujetos.

Los 28 sujetos fueron evaluados con imágenes de RM, que incluían una secuencia volumétrica con ponderación T1 sin contraste endovenoso (3D Fast Spoiled Gradient Echo, TR $=7$ milisegundos, $\mathrm{TE}=2,8$ milisegundos, $\mathrm{TI}=400$ milisegundos, flip angle $=11^{\circ}$, número de excitaciones $=1$, field of view $=$ $16 \times 16 \mathrm{~cm}$, matriz $=256 \times 256$, espesor de corte $=1,2 \mathrm{~mm}$, espacio entre cortes $=0 \mathrm{~mm}$ ). Los estudios fueron adquiridos en un equipo Signa HDxt 3 Tesla (GE, Milwaukee, WI, EEUU), con bobina de cerebro de 8 canales.

Las secuencias ponderadas en T1 volumétricas obtenidas fueron analizadas en forma subjetiva (inspección visual) y objetiva (segmentaciones manuales y automáticas), con la finalidad de graduar o cuantificar la atrofia de las formaciones hipocampales.

El análisis subjetivo fue realizado en forma independiente por 10 médicos especialistas en diagnóstico por imágenes de nuestra institución. Los operadores fueron divididos en 5 grupos según los años de experiencia; grupo 1: menos de 2 años de experiencia, grupo 2: 2 a 4 años de experiencia, grupo 3: 4 a 6 años de experiencia, grupo 4: 6 a 10 años de experiencia, grupo 5: más de 10 años de experiencia. En un software diseñado a medida dentro de nuestra institución (homologable a otros visualizadores comerciales disponibles), generado con la intención de anonimizar los datos de los sujetos, agilizar la evaluación y dejar registro de los resultados, los operadores inspeccionaron visualmente la secuencia volumétrica ponderada en T1 en los planos axial, coronal y sagital, sin conocimiento del nivel cognitivo ni de la edad de los pacientes, clasificando a los sujetos dentro de los siguientes grupos: atrofia hipocampal nula (0), leve (1), moderada (2) o severa (3). Los resultados fueron comparados con los volúmenes hipocampales totales promedios obtenidos mediante segmentación manual (método de referencia).

El AOM se basó en la segmentación corte a corte en el plano coronal de los hipocampos según el HPHS. Dicha segmentación fue realizada por dos operadores. El protocolo detalla los límites precisos para realizar el procedimiento y posee un módulo de entrenamiento y evaluación. ${ }^{12}$

La segmentación y volumetría automática (AOA), fue realizada por medio del software FreeSurfer 5.3 (http:// surfer.nmr.mgh.harvard.edu/) en un computador hecho a medida (OS: Linux Xubuntu 12.04 LTS x64bits, CPU: Intel Core i7-3820 3.6GHz, GPU: Nvidia GeForce GT520, RAM: 16 $\mathrm{Gb})$. Dicho software es uno de los más utilizados hoy en día en los laboratorios de neuroimágenes para la parcelación y segmentación de las estructuras corticales y subcorticales respectivamente. Los resultados obtenidos por el FreeSurfer fueron evaluados visualmente por dos operadores, para confirmar la correcta segmentación de los hipocampos realizada por el software.

Los resultados fueron analizados con el paquete estadístico $\mathrm{R}$ versión 3.2.4. Se obtuvieron coeficientes de

Tabla 1 Análisis de las diferencias entre grupos de edad, género y volúmenes hipocampales

\begin{tabular}{|l|l|l|l|l|}
\hline & $\begin{array}{l}\text { Control } \\
\text { sano (CN) }\end{array}$ & $\begin{array}{l}\text { Deterioro cognitivo } \\
\text { leve temprano (DCLt) }\end{array}$ & $\begin{array}{l}\text { Deterioro cognitivo leve } \\
\text { avanzado (DCLa) }\end{array}$ & $\begin{array}{l}\text { Enfermedad de } \\
\text { Alzheimer (EA) }\end{array}$ \\
\hline Edad (mediana, rango) ${ }^{1}$ & $67(65-81)$ & $69(68-84)$ & $78(75-81)$ & $75(69-84)$ \\
\hline Género (F:M) & & $3: 4$ & $5: 2$ & $6: 1$ \\
\hline $\begin{array}{l}\text { Volumen hipocampos izquierdos } \\
\text { (promedio } \pm \text { DS) }\end{array}$ & $3,73 \pm 0,55$ & $3,43 \pm 0,50$ & $2,85 \pm 0,54$ & $2,36 \pm 0,71$ \\
\hline Volumen hipocampos derechos $^{4}$ & $3,86 \pm 0,47$ & $3,31 \pm 0,49$ & $2,76 \pm 0,45$ & $2,32 \pm 0,53$ \\
\hline
\end{tabular}

${ }^{1}$ Las diferencias de edad entre grupos fueron analizadas por ANOVA, seguidos de Student's $t$-test, siendo la diferencia de edad significativa entre CN y $\operatorname{DCLt}(p=0,005)$.

${ }^{2}$ Se analizaron las diferencias entre las proporciones de género utilizando el test exacto de Fisher, sin hallarse diferencias significativas.

${ }^{3}$ Las diferencias de volumen hipocampal izquierdo entre grupos fueron analizadas por ANOVA, seguidos de Student's $t$-test, siendo la diferencia de volumen significativa entre CN versus EA $(p=0,002)$; CN vs DCLa $(p=0,01)$; DCLt vs EA $(p=0,007)$.

${ }^{4}$ Las diferencias de volumen hipocampal derecho entre grupos fueron analizadas por ANOVA, seguidos de Student's $t$-test, siendo la diferencia de volumen significativa entre CN versus EA ( $p=0,0001)$; CN vs DCLa $(p=0,0008)$; DCLt vs EA $(p=0,004)$. Para los cálculos de Student's t-test se usó la corrección de Bonferroni, siendo el valor de P significativo de 0,013 . 
correlación rho de Spearman para las variables discretas y correlación intraclase para las variables continuas. Los valores de $p$ se consideraron significativos por debajo de 0,05 para las correlaciones de Spearman aplicando la corrección de Bonferroni y por debajo de 0,05 para las correlaciones intraclase.

\section{Resultados}

Análisis Subjetivo Visual: Se obtuvieron coeficientes de correlación rho de Spearman para cada evaluador y en forma conjunta, confrontando las evaluaciones visuales con los resultados promedio de las volumetrías manuales. Los coeficientes de correlación de Spearman individuales y sus intervalos de confianza del 95\% (IC 95\%) fueron de $-0,47$ (IC $95 \%-0,80--0,13),-0,52(-0,83--0,21),-0,53(-0,82--0,24)$, $-0,61(-0,86--0,35),-0,62(-0,89--0,35),-0,63(0,89--0,38),-$ $0,74(-0,92--0,57),-0,78(-0,96--0,60),-0,79(0,95--0,64)$ y $-0,80(-0,97--0,64)$. El coeficiente de correlación de Spearman del promedio de todos los evaluadores en forma conjunta fue de -0,81 (IC 95\%-0,96--0,66) (-Fig. 1).

Análisis Objetivo Manual: Se obtuvieron valores en $\mathrm{mm}^{3}$ para cada uno de los hipocampos de los 28 individuos evaluados por dos operadores (-Fig. 2). Se obtuvieron coeficientes de correlación intraclase (ICC) entre los dos operadores que realizaron las segmentaciones manuales, siendo de $0,88(p<0,0001)$ para los hipocampos izquierdos y de $0,86(p<0,0001)$ para los hipocampos derechos (-Figs. 3 A y B).

Análisis Objetivo Automático: Se obtuvieron los valores en $\mathrm{mm}^{3}$ para cada uno de los hipocampos de los 28 individuos (-Fig. 4). Se obtuvieron coeficientes de correlación intraclase para cada hipocampo, confrontando los resultados de la segmentación automática contra las segmentaciones manuales realizadas por los dos operadores en forma independiente, siendo esos de 0,63 $(p<0,0001)$ y $0,44(p<0,0001)$ para los hipocampos izquierdos y de $0,66(p<0,0001)$ y $0,55(p<0,0001)$ para los hipocampos derechos.

También se obtuvieron coeficientes de correlación intraclase para cada hipocampo, confrontando los resultados de la segmentación automática contra el promedio de las segmentaciones manuales realizadas por ambos operadores, siendo esos de $0,54(p<0,0001)$ para los hipocampos izquierdos (-Fig. 5A) y de 0,61 $(p<0,0001)$ para los hipocampos derechos (-Fig. 5B).

\section{Discusión}

Una de las primeras manifestaciones de la EA y el DCL es el trastorno en la memoria a corto plazo. Las formaciones hipocampales (y sus vías de conexión), son los sustratos anatómicos principales que están involucrados en la generación y conservación de la memoria. ${ }^{13}$ La pérdida neuronal a nivel de los hipocampos se verá reflejada como atrofia. Ella puede ser evaluada y medida por diferentes métodos de imágenes, así como en estudios post-mortem.

La determinación del grado de atrofia hipocampal es uno de los principales marcadores que ha sido históricamente utilizado para evaluar a los pacientes con EA. En la actualidad, las imágenes diagnósticas, particularmente la $\mathrm{RM}$, nos permiten evaluar el grado de atrofia in vivo en pacientes con EA manifiesta o estadios clínicos tempranos. $^{14}$ Frente a la diversidad de métodos para

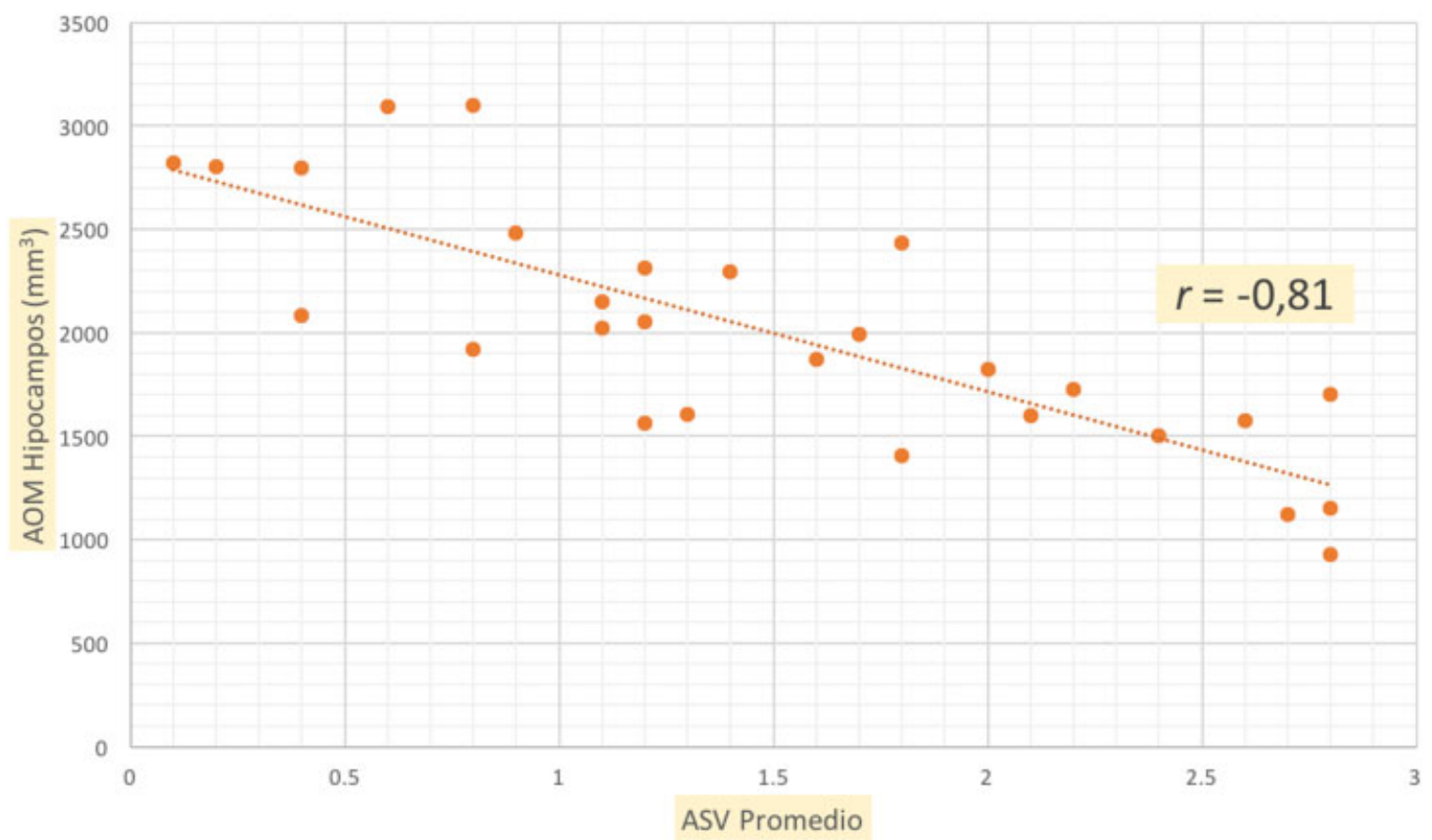

Fig. 1 Correlación de Spearman del valor promedio de los análisis subjetivos visuales (ASV) de todos los evaluadores contra el análisis objetivo manual (AOM) promedio de ambos hipocampos. 

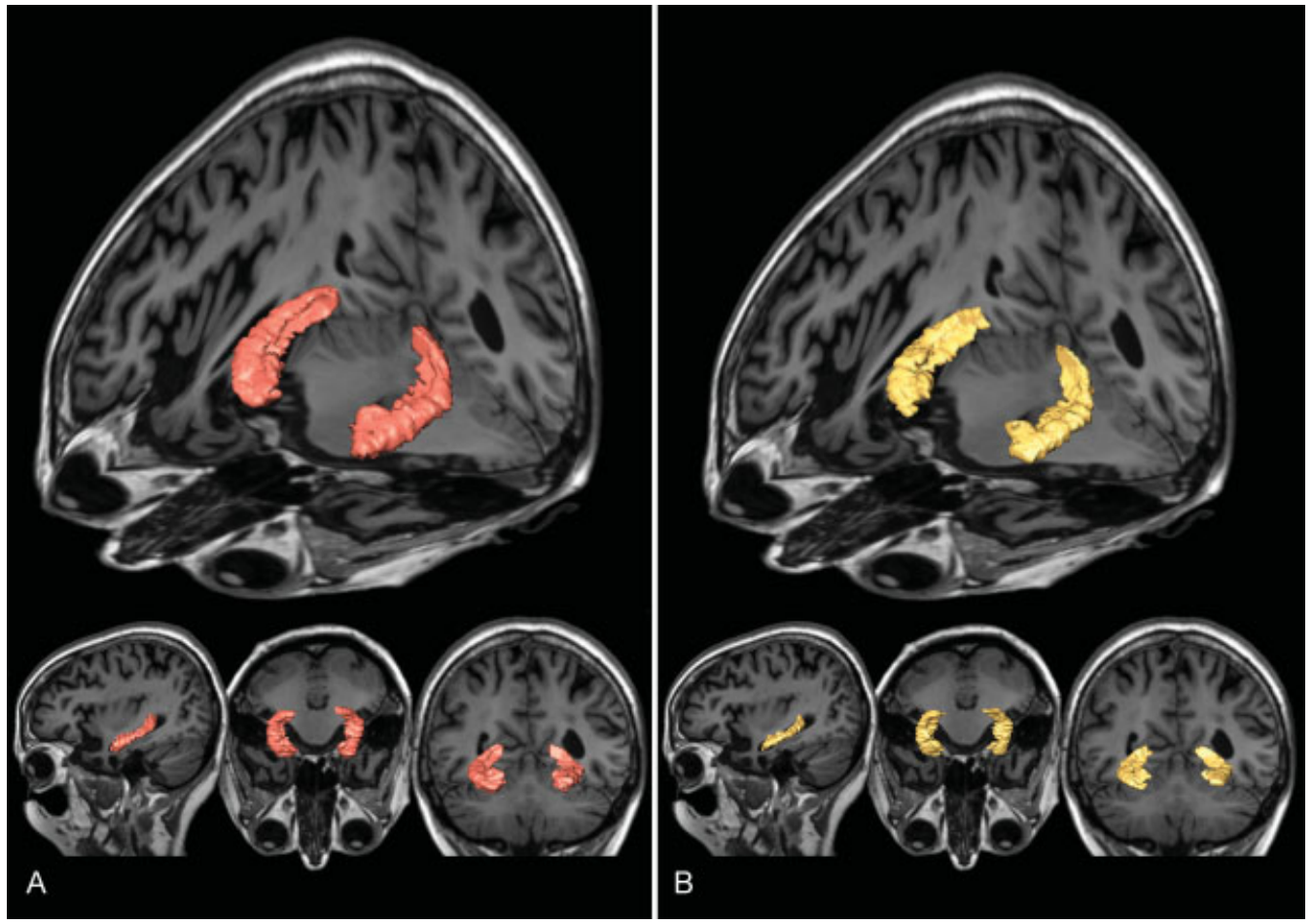

Fig. 2 AOM de los hipocampos de un sujeto realizado por los dos operadores en rojo (A) y amarillo (B). Se observan las reconstrucciones volumétricas de las formaciones hipocampales, superpuestas a una secuencia volumétrica ponderada en $\mathrm{T} 1$ en posición oblicua (imagen superior) y en posiciones sagital, axial y coronal (imágenes inferiores).
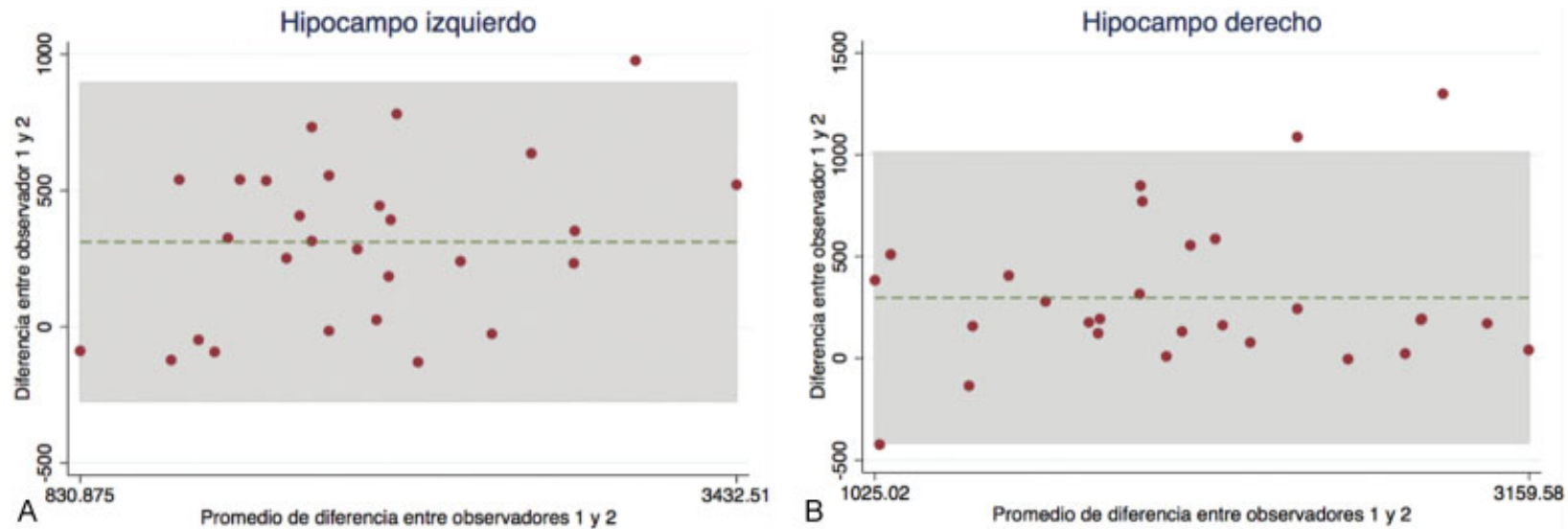

Fig. 3 Gráficos de Bland-Altman representando los coeficientes de correlación intraclase entre operadores que realizaron el AOM de los hipocampos izquierdos (A) y derechos (B).

cuantificar la atrofia, nuestro trabajo evaluó algunos de ellos, realizando un análisis comparativo de los mismos.

Si bien los métodos subjetivos o semi-objetivos han demostrado cierta utilidad en la práctica clínica, su confiabilidad es siempre inferior y no logra aproximarse a la volumetría manual. ${ }^{15}$ Nuestro trabajo también demostró una gran variabilidad entre los resultados de los diferentes operadores al realizar el ASV, obteniéndose coeficientes de correlación de Spearman individuales de entre -0,47 y -0,80. Se demostró una leve mejoría en la correlación con los resultados del AOM al comparar los resultados de evaluadores con pocos o muchos años de experiencia. Sin embargo, se obtiene una clara mejoría en el coeficiente de correlación realizando un promedio de las graduaciones realizadas por todos los evaluadores siendo este de $-0,81$. Debe destacarse que esas correlaciones son negativas debido a que a menor volumen hipocampal determinado por AOM, es esperable que el valor numérico de atrofia asignado por ASV $(0=$ nula, $1=$ leve, $2=$ moderada y $3=$ severa $)$ sea mayor.

La segmentación manual se ha postulado como el método de referencia. Diversos autores y grupos de trabajo han publicado manuales definiendo dónde se deberían trazar los límites hipocampales. Una vez definidos los límites, el principal inconveniente de la segmentación manual es el tiempo requerido al operador, sobre todo en la práctica clínica. El tiempo de segmentación promedio requerido 


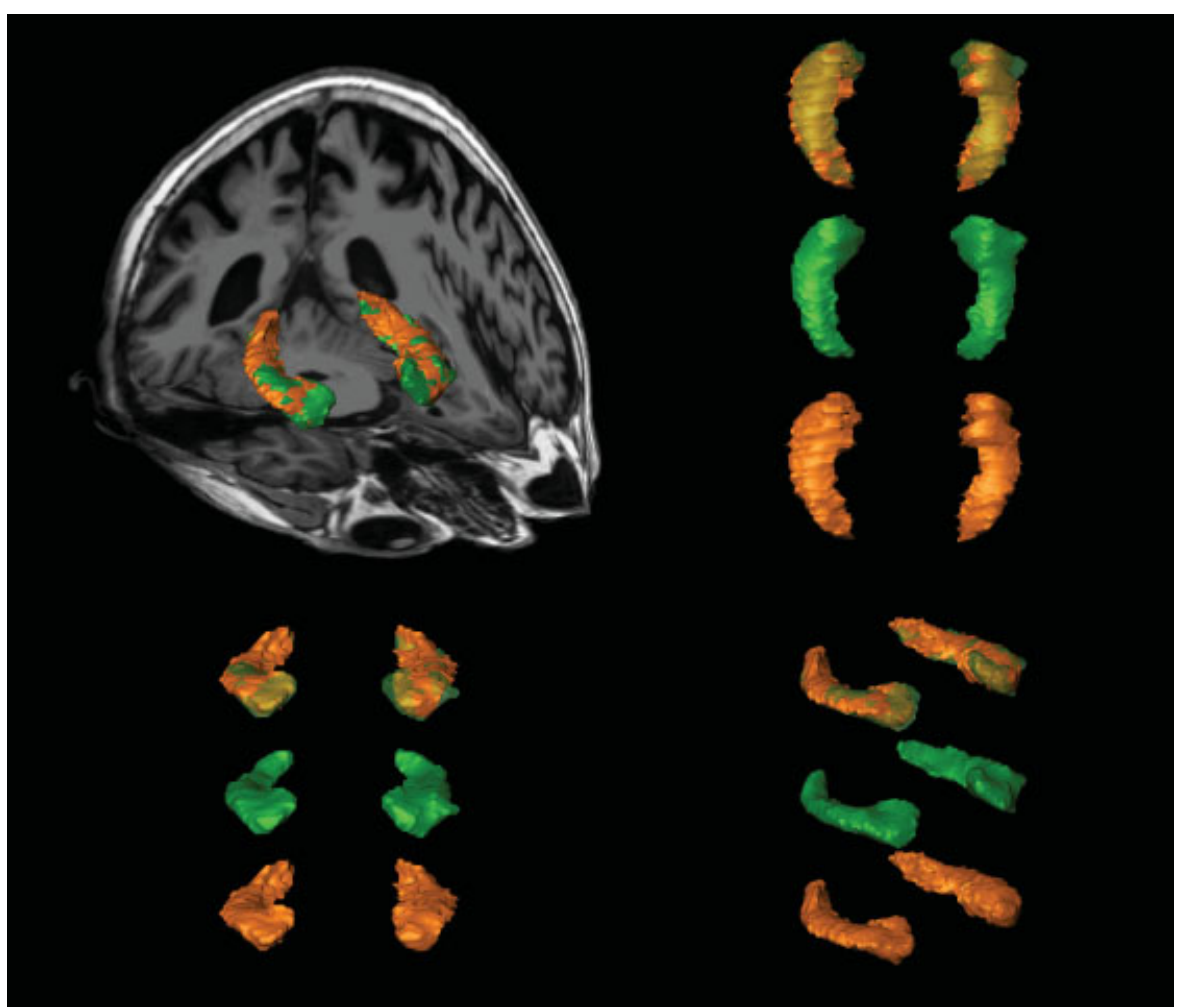

Fig. 4 AOM de los hipocampos de un sujeto vs análisis objetivo automático (AOA). Se observan las reconstrucciones volumétricas de las segmentaciones manuales (en verde), automáticas (en naranja) y sus fusiones (en verde-naranja) de las formaciones hipocampales. En la esquina superior-izquierda, en una vista oblicua superpuestas a una secuencia volumétrica ponderada en T1, en la esquina superior-derecha en una vista superior, en la esquina inferior-izquierda en una vista anterior y en la esquina inferior-derecha en una vista oblicua.
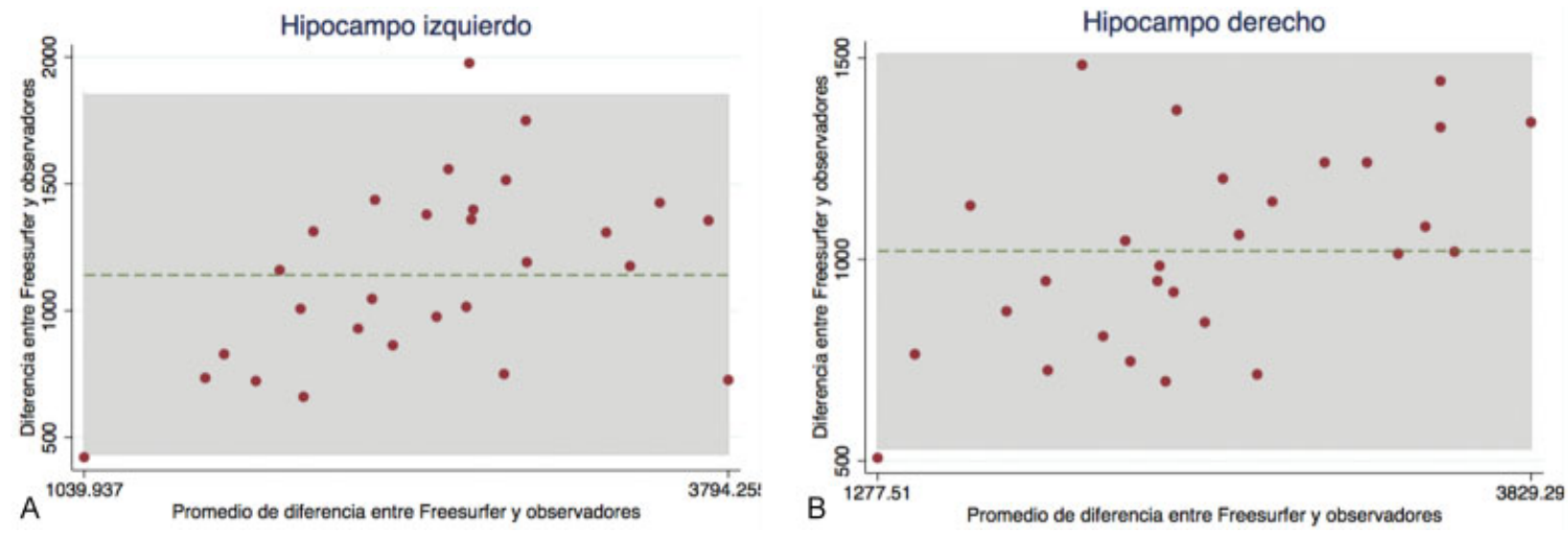

Fig. 5 Gráficos de Bland-Altman representando los coeficientes de correlación intraclase entre el AOM promedio de los hipocampos izquierdos (A) y derechos (B) contra los valores obtenidos por AOA.

para cada operador fue de aproximadamente 10 minutos por hipocampo. Se obtuvieron buenos coeficientes de correlación inter-observador $(0,86$ y 0,88$)$.

Actualmente incluso se está trabajando en la unificación de los métodos de subsegmentación de la formación hipocampal tanto mediante procesos manuales como automáticos, permitiendo diferenciar los volúmenes de sus componentes (giro dentado, asta de Amón, subiculum) y su relación específica con la EA así como con otras patologías. ${ }^{16}$

Los métodos de segmentación automática poseen la ventaja de no requerir gran cantidad de tiempo de un operador humano, si bien requieren prolongados tiempos de procesamiento analítico informático. En nuestra experiencia, utilizando el software FreeSurfer 5.3, requirió de aproximadamente 474 minutos +/- 48 minutos (media de 8 horas), para obtener la volumetría de los hipocampos (entre otros resultados de procesos no independientes como parcelación cortical y segmentación de estructuras subcorticales). El tiempo de operador humano requerido es mínimo para la carga de datos y supervisión de resultados. Nuestros resultados arrojan una moderada correlación entre el AOM y AOA, siendo esos de entre 0,54 y 0,61. Este software está continuamente en desarrollo, 
mejorando los resultados obtenidos a lo largo de las versiones que se han ido distribuyendo. ${ }^{17}$

Como perspectiva a futuro se menciona que, si bien la atrofia hipocampal es un marcador sensible, no permite tamizar los pacientes en estadios preclínicos de la EA, por lo que sería de utilidad validar biomarcadores por neuroimágenes (tanto en RM como en PET) que permitan realizar el diagnóstico en fases tempranas de la enfermedad y con mayor especificidad. ${ }^{18,19}$

\section{Conclusión}

El presente trabajo demuestra que la volumetría automática (AOA), tiene una correlación moderada con el método de referencia (AOM) para la evaluación de la atrofia hipocampal por RM. Por lo tanto, deberían tomarse recaudos antes de implementar esa metodología en servicios de neuroradiología en pacientes con deterioro cognitivo y sospecha de EA.

Si bien el ASV demostró correlaciones individuales disimiles con el AOM, el promedio de dichas evaluaciones sigue siendo superior al AOA.

\section{Colaboradores}

Tatiana Escobar, Laura Falcón, Romina Goñi, Gabriela Michelin, Pablo Pfister, Diego Pineda y Francisco Sepúlveda Hermosilla.

\section{Responsabilidades Éticas}

Protección de personas y animales. Los autores declaran que para esta investigación no se han realizado experimentos en seres humanos ni en animales.

Confidencialidad de los datos. Los autores declaran que en este artículo no aparecen datos de pacientes.

Derecho a la privacidad y consentimiento informado. Los autores declaran que en este artículo no aparecen datos de pacientes.

\section{Conflicto de Intereses}

Los autores declaran no tener ningún conflicto de intereses, excepto el Dr. Chaves que declara como posible conflicto de interés ser consultor de la Revista Argentina de Radiología y el Dr. Ventrice que declara como posible conflicto de interés ser co-autor de un trabajo citado.

\section{Agradecimientos}

A nuestros colaboradores: Tatiana Escobar, Laura Falcón, Romina Goñi, Gabriela Michelin, Pablo Pfister, Diego Pineda y Francisco Sepúlveda Hermosilla.

\section{Bibliografía}

1 Dubois B, Feldman HH, Jacova C, et al. Research criteria for the diagnosis of Alzheimer's disease: revising the NINCDS-ADRDA criteria. Lancet Neurol 2007;6(08):734-746

2 van de Pol LA, Hensel A, Barkhof F, Gertz HJ, Scheltens P, van der Flier WM. Hippocampal atrophy in Alzheimer disease: age matters. Neurology 2006;66(02):236-238
3 Scheltens P, Leys D, Barkhof F, et al. Atrophy of medial temporal lobes on MRI in "probable" Alzheimer's disease and normal ageing: diagnostic value and neuropsychological correlates. J Neurol Neurosurg Psychiatry 1992;55(10):967-972

4 Bobinski M, de Leon MJ, Wegiel J, et al. The histological validation of post mortem magnetic resonance imaging-determined hippocampal volume in Alzheimer's disease. Neuroscience 2000;95(03):721-725

5 Boccardi M, Bocchetta M, Apostolova LG, et al; EADC-ADNI Working Group on the Harmonized Protocol for Manual Hippocampal Segmentation. Delphi definition of the EADCADNI Harmonized Protocol for hippocampal segmentation on magnetic resonance. Alzheimers Dement 2015;11(02):126-138

6 Bocchetta M, Boccardi M, Ganzola R, et al; EADC-ADNI Working Group on The Harmonized Protocol for Manual Hippocampal Segmentation and for the Alzheimer's Disease Neuroimaging Initiative. Harmonized benchmark labels of the hippocampus on magnetic resonance: the EADC-ADNI project. Alzheimers Dement 2015;11(02):151-60.e5

7 Frisoni GB, Jack CR Jr, Bocchetta M, et al; EADC-ADNI Working Group on The Harmonized Protocol for Manual Hippocampal Volumetry and for the Alzheimer's Disease Neuroimaging Initiative. The EADC-ADNI Harmonized Protocol for manual hippocampal segmentation on magnetic resonance: evidence of validity. Alzheimers Dement 2015;11(02):111-125

8 Dill V, Franco AR, Pinho MS. Automated methods for hippocampus segmentation: the evolution and a review of the state of the art. Neuroinformatics 2015;13(02):133-150

9 Morey RA, Petty CM, Xu Y, et al. A comparison of automated segmentation and manual tracing for quantifying hippocampal and amygdala volumes. Neuroimage 2009;45(03):855-866

10 Chupin M, Hammers A, Liu RSN, et al. Automatic segmentation of the hippocampus and the amygdala driven by hybrid constraints: method and validation. Neuroimage 2009;46(03):749-761

11 Russo MJ, Gustafson D, Vázquez S, et al; Argentina-Alzheimer's Disease Neuroimaging Initiative. Creation of the ArgentinaAlzheimer's Disease Neuroimaging Initiative. Alzheimers Dement 2014;10(1, Suppl):S84-S87

12 Duchesne S, Valdivia F, Robitaille N, et al; EADC-ADNI Working Group on The Harmonized Protocol for Manual Hippocampal Segmentation and for the Alzheimer's Disease Neuroimaging Initiative. Manual segmentation qualification platform for the EADC-ADNI harmonized protocol for hippocampal segmentation project. Alzheimers Dement 2015;11(02):161-174

13 Bartsch T, Wulff P. The hippocampus in aging and disease: From plasticity to vulnerability. Neuroscience 2015;309:1-16

14 Schröder J, Pantel J. Neuroimaging of hippocampal atrophy in early recognition of Alzheimer's disease-a critical appraisal after two decades of research. Psychiatry Res 2016;247:71-78

15 Clerx L, van Rossum IA, Burns L, et al. Measurements of medial temporal lobe atrophy for prediction of Alzheimer's disease in subjects with mild cognitive impairment. Neurobiol Aging 2013; 34(08):2003-2013

16 Yushkevich PA, Amaral RS, Augustinack JC, et al; Hippocampal Subfields Group (HSG). Quantitative comparison of 21 protocols for labeling hippocampal subfields and parahippocampal subregions in in vivo MRI: towards a harmonized segmentation protocol. Neuroimage 2015;111:526-541

17 Clerx L, Gronenschild EH, Echavarri C, Verhey F, Aalten P, Jacobs HI. Can FreeSurfer Compete with Manual Volumetric Measurements in Alzheimer's Disease? Curr Alzheimer Res 2015;12(04):358-367

18 Berti V, Polito C, Lombardi G, Ferrari C, Sorbi S, Pupi A. Rethinking on the concept of biomarkers in preclinical Alzheimer's disease. Neurol Sci 2016;37(05):663-672

19 Besson FL, La Joie R, Doeuvre L, et al. Cognitive and Brain Profiles Associated with Current Neuroimaging Biomarkers of Preclinical Alzheimer's Disease. J Neurosci 2015;35(29):10402-10411 